\title{
Investigation of New Algorithms for Estimation of Losses in Microwave Devices Based on a Waveguide or a Meander Line
}

\author{
D. Plonis*, A. Katkevičius, V. Mališauskas, A. Serackis and D. Matuzevičius \\ Vilnius Gediminas Technical University, Department of Electronic System, \\ Naugarduko Str. 41-413, LT-03227, Vilnius, Lithuania
}

(Received November 19, 2015; in final form February 19, 2016)

\begin{abstract}
The aim of this paper was to introduce the estimation of the losses in the prototypes of the microwave devices. We proposed in this paper two algorithms: an algorithm for evaluation of losses in the microwave devices based on a waveguide and an algorithm for evaluation of losses in the microwave devices based on a meander line. In order to verify the results of losses evaluation for the waveguide-based device, we have used the electrodynamic model of the open cylindrical gyrotropic waveguide with one anisotropic dielectric layer and the electrodynamic model of the open cylindrical gyrotropic waveguide with two dielectric layers. In order to verify the results of losses evaluation for the meander line-based device, we have used the model of the meander line with asymmetrical periodical inhomogeneity. The comparison of the losses evaluation results received by the algorithms proposed in this paper and the results received by application of the commercial software together with the alternative methods confirmed that the proposed algorithms produced correct results.
\end{abstract}

DOI: 10.12693/APhysPolA.129.414

PACS/topics: 84.40.Az

\section{Introduction}

Waveguides and meander lines are widely used in design of travelling-wave tubes, delay lines, phase modulators or converters, transmitters and other microwave devices [1-5]. The methods for microwave device design, models and properties of various types of waveguides and meander line based systems have been already analysed in the textbooks [5], scientific papers [6-8] and monographs [9]. However, the design of new microwave devices, based on a new combination of the waveguide materials and the design of microwave devices based on the new shape of the meander line requires additional investigation of the losses received in the newly designed system.

In order to estimate the losses in the newly designed microwave device, usually the simplified model of the device is prototyped and used in the experimental tests. Although, frequently the ideal models of microwave systems are considered disregarding the attenuation caused by the material used in the design of the device.

The gyrotropic waveguides are usually used to build a polarizer based on a Faraday effect. Erickson discussed an example of such polarizer, which was designed to work in $74-110 \mathrm{GHz}$ frequency range [10]. In addition, we may find axially magnetized cylindrical phase shifters, investigated by Iqbal and Gibson, which are based on the semiconductor waveguides $[11,12]$. However, the differential phase shift received for these microwave devices is

*corresponding author; e-mail: darius.plonis@vgtu.lt only 90 degrees and given papers lacks the more detailed analysis of the frequency characteristics for the proposed phase shifters. Iqbal and Gibson presented only the analysis of the $\mathrm{TE}_{11}$ mode propagation in the proposed phase shifter model. Moreover, no analysis of the attenuation was given for these phase shifters.

It is already known that the characteristics of devices designed using gyroelectric (semiconductor) materials can be controlled by changing the temperature, the longitudinal and axial magnetic flux density, the density of the free carriers and $\gamma$-rays. It is also known that the high density of electrons in the material increases the attenuation of electromagnetic wave in the waveguides (also called magneto-active waveguides). These features limits the application of the given materials for the transmission of the EM wave $[9,13]$.

In order to design a waveguide based on a dielectricmetamaterial, Huang introduced the new models of waveguides with lower attenuation [14]. Huang and Zhang proposed to apply the additional layers, made from anisotropic cladding using silver and dielectric rings $[14,15]$. The investigation of different alternative waveguide structures (closed, open multi-layered) with an external layer, made from the perfect conductor also can be found in the literature [16-18]. However, the use of dielectric material for an external layer was not analysed in detail.

Nickelson et al. already performed the investigation of the cut-off frequencies for the gyroelectric semiconductor based waveguide [19]. However, they did not estimate the attenuation of the EM wave in these waveguides. In the following works, they have estimated the attenuation characteristics (together with dispersion 
characteristics) of the open cylindrical, rectangular $\mathrm{SiC}$ and metamaterial waveguides [20-22]. The attenuation estimated for $\mathrm{SiC}$ waveguides was very high. The scattering of the EM waves also appeared in dielectric and metamaterial cylindrical structures. Bucinskas et al. and Oraizi et al. have published the results of the analysis of these processes [23-25].

Kelebekler et al. used the method of moment (MoM) for the analysis of the open cylindrical gyroelectric waveguides. The electrodynamical models of waveguide with plasma and without external dielectric layer were used [26, 27]. Liu et al. analysed circular conducting waveguides, filled with the gyroelectric material, using dyadic Green's functions [28]. However the attenuation of the electromagnetic wave were not analysed in these papers.

Another type of microwave devices analysed in this paper is based on a meander line. Djurič et al. proposed an inductive displacement sensor based on a meander structure [1]. Golanka proposed a sensor composed from two sensor elements with meander-type inductive coils [29]. It have been showed that it is possible to detect the normal and the tangential displacement by using these two sensors elements. The technology and few applications of low temperature ceramic-based sensors using meander structures are presented in the Golanka paper [29].

Meander systems are widely used in the antenna design. The meandering is one of the methods used to reduce the size of the antenna [30]. The meander linebased antennas are usually designed using empirically estimated relations or using numerical methods. The dimensions of the couplers can be reduced using meandering circuit lines in the DVB-T/H transmitter system design [6].

A new meander-type laminated coupled structure was proposed in order to solve the problem of low transporting capacity and efficiency of the typical capacitive coupled power transfer system. The equivalent circuit model and results of the simulation analysis showed that the received output power of the system with the new structure is twice higher than the one received using the traditional type of the power transfer system [8].

Meander structures are also used in delay systems which are widely used in $\mathrm{rf}$ and microwave electronics: travelling-wave tubes for velocity matching of flight of the electron beam and electromagnetic waves, in antenna arrays to form a predetermined pattern and control it. Gurskas et al. proposed to perform an investigation of the dispersion of phase delay in the meander lines using hybrid technique [31].

The wideband microwave phase shifters based on various structures of meander lines may provide a wide bandwidth from $5.5 \mathrm{GHz}$ to $18 \mathrm{GHz}[32,33]$. These phase shifters offer low insertion loss and low values of the reflection coefficients for the same frequency range. However, the design of this type of phase shifters becomes complicated for the higher frequencies, such as $90 \mathrm{GHz}$ or $100 \mathrm{GHz}$ due to the small dimensions of the line.
In addition, the shift of the phase angles higher than 180 degrees is followed by a high attenuation. Therefore, the prototype of the phase shifter should be additionally analysed in order to estimate the influence of the high electromagnetic attenuation to the parameters of the meander line. However, most of the articles discussed above presents the analysis of the ideal systems without taking into account the losses in the materials used for the designed systems.

In this paper, we propose two algorithms for modelling of waveguide-based or meander line-based systems. The proposed algorithms allows assessing the influence of the losses to the frequency characteristics of designed microwave system.

\section{Materials and methods}

We have designed the algorithms proposed in this paper for the estimation of the losses in the microwave systems. We focused on the cylindrical gyrotropic waveguides and the meander systems. We performed the detailed analysis on the open cylindrical waveguide with one anisotropic dielectric layer; the open cylindrical waveguide with two dielectric layers and the meander delay line with asymmetrical periodical inhomogeneities.

\subsection{The models of the open cylindrical waveguides}

Before the investigation of the algorithm for the analysis of losses in the open cylindrical waveguide-based microwave device, we understood the importance to investigate the relations between the electrodynamical parameters of the waveguide and the analysis methods used for estimation of device characteristics. We have designed a universal algorithm, which can be used for the analysis of the parameters of the waveguides shown in Fig. 1 and Fig. 2. In the whole text underlining means complex values.

Maxwell's complex differential equations, coupled mode and boundary condition methods are used to obtain the complex dispersion equation.

The first area of models (Figs. 1 and 2) is the same and the complex elements which describe EM waves propagation in this area are presented in [9]. The longitudinal components of electric and magnetic fields in gyrotropic core are $\underline{E}_{z}^{\mathrm{g}}$ and $\underline{H}_{z}^{\mathrm{g}}$, respectively.

The longitudinal components of electric and magnetic fields, which satisfies Maxwell's equations in anisotropic dielectric layer (area 2) of the model (Fig. 1), can be presented as

$$
\begin{aligned}
& \underline{E}_{z}^{\mathrm{ad}}=\left[\underline{A}_{2} J_{m}\left(\underline{k}_{\perp 1}^{\mathrm{ad}} r^{\mathrm{g}}\right)+\underline{A}_{3} N_{m}\left(\underline{k}_{\perp 1}^{\mathrm{ad}} r^{\mathrm{g}}\right)\right] \mathrm{e}^{\mathrm{j} \varphi}, \\
& \underline{H}_{z}^{\mathrm{ad}}=\left[\underline{B}_{2} J_{m}\left(\underline{k}_{\perp 2}^{\mathrm{ad}} r^{\mathrm{g}}\right)+\underline{B}_{3} N_{m}\left(\underline{k}_{\perp 2}^{\mathrm{ad}} r^{\mathrm{g}}\right)\right] \mathrm{e}^{\mathrm{j} \varphi},
\end{aligned}
$$

where $\underline{A}_{2,3}$ and $\underline{B}_{2,3}$ are unknown amplitude coefficients; $J_{m}\left(\underline{k}_{\perp 1,2}^{\mathrm{ad}} r^{\mathrm{g}}\right)$ is the Bessel function of the first kind of the $m$-th order with the complex argument $\underline{k}_{\perp 1,2}^{\text {ad }} r^{\mathrm{g}}$; $N_{m}\left(\underline{k}_{\perp 1,2}^{\mathrm{ad}} r^{\mathrm{g}}\right)$ is the Bessel (Neumann) function of the second kind of the $m$-th order with the complex argument; 

1. $\stackrel{\leftrightarrow}{\mu}_{\mathrm{r}}^{\mathrm{f}}, \underline{\varepsilon}_{\mathrm{r}}^{\mathrm{f}}$
$\underline{\mu}_{\mathrm{r}}^{\mathrm{s}}, \quad \underline{\varepsilon}_{\mathrm{r}}$
2. $\mu_{\mathrm{r}}^{\mathrm{ad}}, \stackrel{\vec{\varepsilon}}{\mathrm{r}}_{\mathrm{ad}}$
3. $\mu_{\mathrm{r}}^{\mathrm{a}}, \quad \varepsilon_{\mathrm{r}}^{\mathrm{a}}$
$r^{\mathrm{g}}=r^{\mathrm{f}}=r^{\mathrm{s}}$

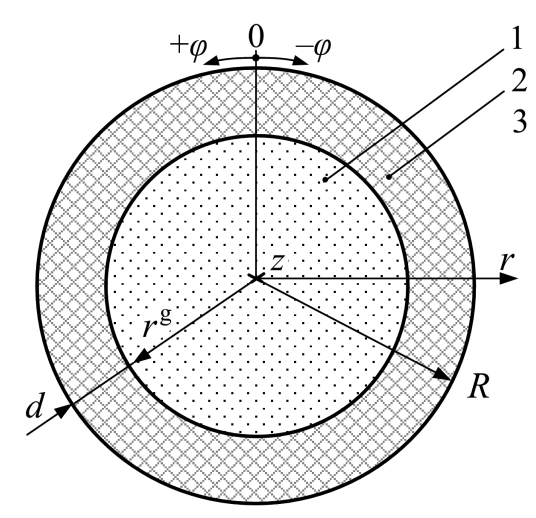

Fig. 1. The electrodynamic model of the open cylindrical gyrotropic waveguide with anisotropic dielectric layer, where: $\stackrel{\leftrightarrow}{\mu}_{\mathrm{r}}^{\mathrm{f}}$ is the complex permeability tensor of gyrotropic core; $\underline{\varepsilon}_{\mathrm{r}}^{\mathrm{f}}$ is the complex permittivity of gyrotropic core; $\stackrel{\leftrightarrow}{\varepsilon}_{\mathrm{r}}^{\mathrm{s}}$ is the complex permittivity tensor of gyrotropic core; $\underline{\mu}_{\mathrm{r}}^{\mathrm{s}}$ is the complex permeability of gyrotropic core; $\mu_{\mathrm{r}}^{\mathrm{ad}}$ is the permeability of anisotropic dielectric layer; $\stackrel{\leftrightarrow}{\varepsilon}_{\mathrm{r}}^{\mathrm{ad}}$ is the complex permittivity tensor of anisotropic dielectric layer; $\mu_{\mathrm{r}}^{\mathrm{a}}$ is the air permeability; $\varepsilon_{\mathrm{r}}^{\mathrm{a}}$ is the air permittivity; $\boldsymbol{B}_{0}$ is the vector of magnetic flux density; $R$ is the radius of the waveguide, $d$ is the width of the anisotropic dielectric layer and $r^{\mathrm{g}}$ is the radius of gyrotropic core.

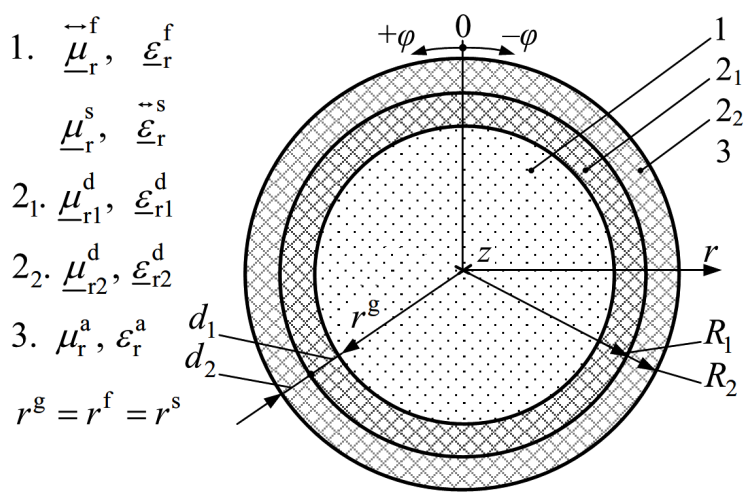

\section{$\otimes$} $\boldsymbol{B}_{0}$

Fig. 2. The electrodynamic model of open cylindrical gyrotropic waveguide with two dielectric layers, where: $\underline{\mu}_{\mathrm{r} 1}^{\mathrm{d}}$ is the complex permeability of first dielectric layer; $\underline{\varepsilon}_{\mathrm{r} 1}^{\mathrm{d}}$ is the complex permittivity of first dielectric layer; $\underline{\mu}_{\mathrm{r} 2}^{\mathrm{d}}$ is the complex permeability of second dielectric layer; $\underline{\varepsilon}_{\mathrm{r} 2}^{\mathrm{d}}$ is the complex permittivity of the second dielectric layer; $R_{1}$ and $R_{2}$ are the radii of the dielectric layers, $d_{1}$ and $d_{2}$ are the widths of the dielectric layers.

$\underline{k}_{\perp 1,2}^{\mathrm{ad}}$ are the transversal wave numbers in anisotropic dielectric layer; $m$ is hybrid mode first (azimuthal) index, describing longitudinal wave constant component by the azimuthal perimeter coordinate $\varphi, j=\sqrt{-1}$ is the complex number.

The wave's transversal numbers in anisotropic (isotropic) dielectric layer can be presented as

$$
\begin{aligned}
& \underline{k}_{\perp 1}^{\mathrm{ad}}=\sqrt{k^{2} \underline{\varepsilon}_{x x}^{\mathrm{ad}}-\underline{\gamma}^{2}}, \\
& \underline{k}_{\perp 2}^{\mathrm{ad}}=\sqrt{\frac{\underline{\varepsilon}_{z z}^{\mathrm{ad}}}{\underline{\varepsilon}_{x x}^{\mathrm{ad}}}\left(k^{2} \underline{\varepsilon}_{x x}^{\mathrm{ad}}-\underline{\gamma}^{2}\right)},
\end{aligned}
$$

where $\gamma=\beta-\mathrm{j} \alpha$ is the complex propagation constant (here $\bar{\beta}=\operatorname{Re}(\gamma)=2 \pi / \lambda_{\mathrm{w}}$ is the phase constant and $\lambda_{\mathrm{w}}$ is the wavelength of the waveguide modes; $\alpha=\operatorname{Im}(\gamma)$ is the attenuation constant); $k=\omega / c$ is the wave number in a vacuum; $\underline{\varepsilon}_{x x}^{\text {ad }}$ and $\underline{\varepsilon}_{z z}^{\text {ad }}$ are the anisotropic dielectric layer tensor diagonal elements.

The third area of models (Figs. 1 and 2) is also the same. Complex elements, which describe EM waves propagation in this area, are presented in [9]. The longitudinal components of electric and magnetic fields are $\underline{E}_{z}^{a}$ and $\underline{H}_{z}^{a}$, respectively, in air.

The azimuthal components of electric $\underline{E}_{\varphi}^{\mathrm{g}, \text { ad,a }}$ and magnetic $\underline{H}_{\varphi}^{\mathrm{g}, \text { ad,a }}$ fields can be obtained from longitudinal components $\underline{E}_{z}^{\mathrm{g}, \text { ad,a }}$ and $\underline{H}_{z}^{\mathrm{g}, \mathrm{ad}, \mathrm{a}}$. The complex dispersion equation is obtained by using boundary conditions. The complex dispersion equation of model of the open cylindrical gyrotropic waveguide with anisotropic dielectric layer (Fig. 1) is the 8th order determinant expression $\underline{D}=\operatorname{det}\left(\underline{a}_{j k}\right)=0$, where $j$ is a column and $k$ is a row index of determinant and $\underline{a}_{j k}$ are complex elements of determinant.

By using the same method, the complex dispersion equation of model of open cylindrical gyrotropic waveguide with two dielectric layers can be obtained only in this case when the complex dispersion equation is the 12 th order determinant expression.

It was problematic to analyse and select the materials for the design of the gyrotropic waveguide based device. The choice of ferrite materials is small but these materials are distinguished by a low attenuation of the propagating waves in the microwave devices. A large choice of semiconductors could be classified by the material type, the type of the material conductivity, the density of free carriers, the mobility of the carriers, the effective mass and the relative permittivity.

It is difficult to perform a physical experiment of the waveguide based microwave device. First of all the diameter of the open cylindrical waveguides is very small. Secondly, these waveguides are long. Thirdly, the cores of the waveguides are hard and fragile and finally the density of free carriers should be low. In addition, we were unable to produce physically the adequate samples and the investigation limited to the numerical experiment only.

\subsection{Algorithm for the estimation of losses in the open cylindrical waveguides}

The basis of the algorithm 1 proposed for the analysis of the losses in open cylindrical waveguide-based device (OCWs) is presented in Fig. 3. The main difference of the algorithm proposed in this paper to the algorithms we have presented in our previous works [34] lies in the 


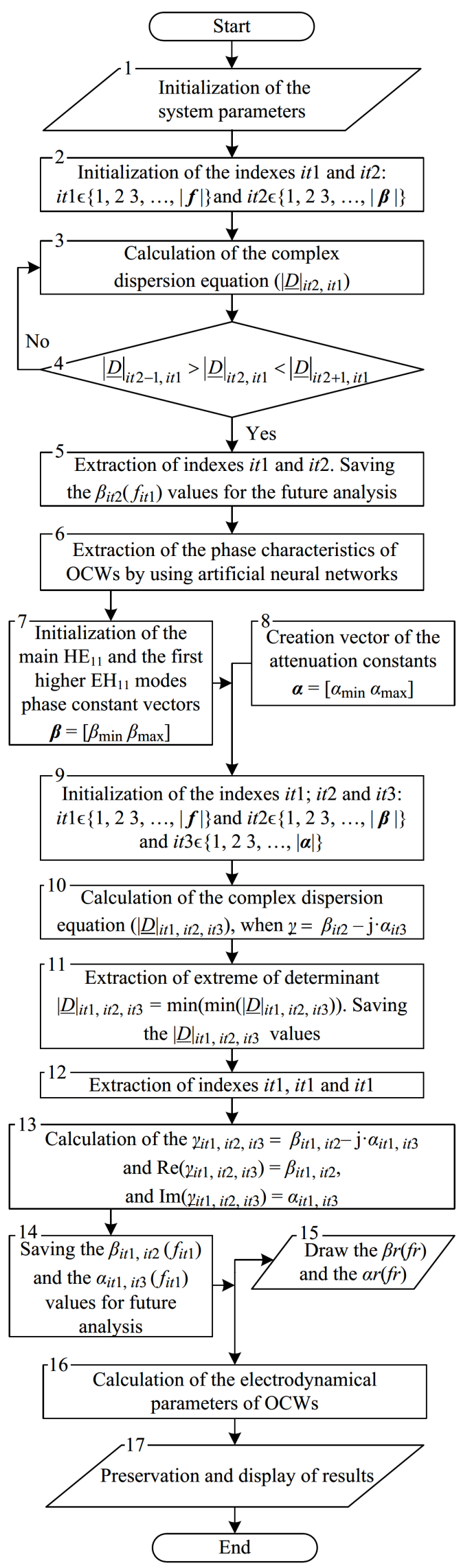

Fig. 3. The basic algorithm for the analysis of the open cylindrical waveguides (algorithm 1 ). calculation of the complex propagation constant of the EM waves, which allows us to evaluate the attenuation of the EM in OCWs.

Firstly, system parameters of OCWs were initialized in the first block for two calculation cases: (1) initialization of the parameters for the OCWs with anisotropic dielectric layer: $\varepsilon_{k}^{s n, p}, N, \mu, r^{\mathrm{g}}, B_{0}, \mu_{\mathrm{r}}^{\mathrm{ad}}, \underline{\varepsilon}_{\mathrm{r}}^{\mathrm{ad}}, H, M_{\mathrm{s}}, m$, $m^{*}, T, \Delta H, \underline{\mu}_{\mathrm{r}}^{\mathrm{s}}, \underline{\varepsilon}_{\mathrm{r}}^{\mathrm{f}}, R, f_{\min }, f_{\max }, \Delta f, \beta_{\min }, \beta_{\max }, \Delta \beta$; (2) initialization of the parameters for the OCWs with two dielectric layers: $\varepsilon_{k}^{\mathrm{s} n, p}, N, \mu, r^{g}, B_{0}, \underline{\mu}_{r 1,2}^{\mathrm{d}}, \underline{\varepsilon}_{r 1,2}^{\mathrm{d}}, H$, $M_{\mathrm{s}}, m, m^{*}, T, \Delta H, \underline{\mu}_{\mathrm{r}}^{\mathrm{s}}, \underline{\varepsilon}_{\mathrm{r}}^{\mathrm{f}}, R_{1,2}, f_{\min }, f_{\max }, \Delta f, \beta_{\min }$, $\beta_{\max }, \Delta \beta$. Here: $\varepsilon_{k}^{\mathrm{s} n, p}$ is the permittivity of semiconductor core; $N$ is the density of free carries; $\mu$ and $m^{*}$ are the mobility and the effective mass of free carries; $H$ is the external magnetic field strength; $\Delta H$ is the width of the resonance line; $M_{\mathrm{s}}$ is the magnetization to saturation. It is very important to select the initial boundaries of EM wave frequencies $f_{\min }$ and $f_{\max }$ and step of EM frequency $-\Delta f$. Selection of the initial boundaries of EM waves phase constants $\beta_{\text {min }}$ and $\beta_{\max }$ and step of EM phase $-\Delta \beta$ is also important.

The initialization of indexes of iterations $i t 1$ and $i t 2$ is performed in the second block. The indexes of iterations depend on lengths of vectors of frequencies $f$ and phase constants $\boldsymbol{\beta}$. The vectors $\boldsymbol{f}$ and $\boldsymbol{\beta}$ consist of values of $f_{\min }, f_{\max }$, and $\Delta f$, and $\beta_{\min }, \beta_{\max }$ and $\Delta \beta$, respectively.

The calculation of the complex dispersion equation $|\underline{D}|$ is performed in the third block. This part of the algorithm consists of the several parts: calculation of the relative complex permittivity and permeability tensors; calculation of the determinant elements $\underline{a}_{j k}$; calculation of the determinant $\underline{D}=\operatorname{det}\left(\underline{a}_{j k}\right)$.

We calculated the phase constants $\beta$ by searching for the global minimum in the solution of the dispersion equation $|\underline{D}|$ (fourth block, see Fig. 3). The extraction of indexes $i t 1$ and $i t 2$ where is the solution of dispersion equation $|\underline{D}|$ is performed in the fifth block. We extracted the phase characteristics of the main and the first higher modes (sixth block, see Fig. 3) by using an algorithm, which was presented in our previous work [34] which is based on artificial neural networks. These extracted characteristics were used for the calculation of the complex propagation constant $\gamma$ and for the future analysis tasks. The initialization of the main $\mathrm{HE}_{11}$ and first higher $\mathrm{EH}_{11}$ modes phase constants $\boldsymbol{\beta}$ vectors and creation $\boldsymbol{\alpha}$ vector of the attenuation constants are performed in 7 and 8 blocks of the algorithm. The vector $\boldsymbol{\alpha}$ consists of values of $\alpha_{\min }, \alpha_{\max }$ and $\Delta \alpha$. The initial boundaries of vector $\boldsymbol{\alpha}$ was $\alpha_{\min }=0,1 / \mathrm{m} ; \alpha_{\max }=5000,1 / \mathrm{m}$ and the step $\Delta \alpha=0.025,1 / \mathrm{m}$. The initialization of new indexes of iterations $i t 1$, it 2 and $i t 3$ is performed in ninth block. The it 3 index of iterations depends on length of vector $\boldsymbol{\alpha}$.

The calculation of the complex dispersion equation $|\underline{D}|$ is performed in the tenth block of the algorithm, when the complex propagation constant depends on the indexes of iterations. The extraction of extreme of 
determinant and indication of locations by indexes it1, it2 and $i t 3$, and calculation of complex propagation constant by indexes $i t 1$, it2 and $i t 3$, and the phase and attenuation constants dependences on the frequency were saved for future analysis and the finally graphical representation of the phase and attenuation constants dependences on the frequency were performed in 11-15 blocks of algorithm (Fig. 3).

The electrodynamical parameters of the OCWs were calculated during sixteenth block of the algorithm. The electrodynamical parameters: cut-off frequencies of modes; central frequency of the OCWs; working frequency range of the OCWs; bandwidth in percentages of the OCWs were calculated by using the same method, presented in our previous work [34].

The differential phase shift module was calculated in seventeenth block of the algorithm 1 by selecting frequency value in the waveguide working frequency range and searching for the intersection of vertical line, drawn at the selected frequency and phase constant characteristics. The differential phase shift module was calculated by using expression

$$
|\Delta \vartheta|_{f=\text { const }}=\left|\left(\beta_{0}-\beta_{B_{0}, T}\right)\right| L 360 / 2 \pi,
$$

where $\beta_{0}$ is the phase constant, when $B_{0}=0$, and $T$ is the initial temperature; $\beta_{B_{0}, T}$ is the phase constant, when $B_{0} \neq 1 \mathrm{~T}$ and $T$ is the higher or lower than initial temperature; $L$ is the length of the OCW.

The attenuation constant dependences on the magnetic flux density (and temperature) were calculated by using similar method to the differential phase shift module calculation. The attenuation constant was calculated by using expression

$$
\left.\alpha L\right|_{f_{=\text {const }}}=20 \log _{10}(\exp (\alpha L)) .
$$

Results are saved in the seventeenth block.

This developed algorithm could be used for the analysis of the simple multilayer dielectric waveguides, gas discharge and dielectric tubes. In addition, it could be adapted for the analysis of the other waveguide models.

There are several important advantages of the first algorithm proposed in this paper (see Fig. 3), comparing to estimation of the waveguide characteristics by commercial software. First advantage is the ability to estimate the attenuation constant dependences on the magnetic flux density, frequency and temperature for any OCWs. Second, is the ability to estimate the bandwidth, which is related to the central frequency, differential phase shift module for OCWs designed from various possible materials (only the main features of the material should be provided). Third, the calculation time, needed to estimate this set of parameters is comparatively short and takes less than 1 min for a OCWs.

\subsection{The meander lines with periodical inhomogeneity}

Simplified models of the ideal meander delay systems without losses were usually used during the analysis of the frequency characteristics of the prototype. The attenuation is always present in the real meander systems.
Frequently it remains unclear what was the influence of the losses caused by attenuation to the frequency characteristics of the meander systems. It was important to create a universal algorithm for performing an analysis of the influence of losses to the frequency characteristics of the meander delay systems.

The model of the meander structure with asymmetrical periodical inhomogeneity or transverse inhomogeneity respect to the longitudinal plane was investigated in order to clarify the influence of losses to the frequency characteristics of the meander delay systems in this particular case. The inhomogeneity in these systems appear due to dielectric holders, bending of the conductor of the meander electrode, errors of manufacturing and for other reasons [36]. A fragment of the asymmetrical meander electrode with different widths of the narrow conductors at the peripheral parts is shown in Fig. 4. This asymmetrical meander electrode has been analysed in order to elucidate whether asymmetrical inhomogeneity affect the width of pass-band of a meander system.

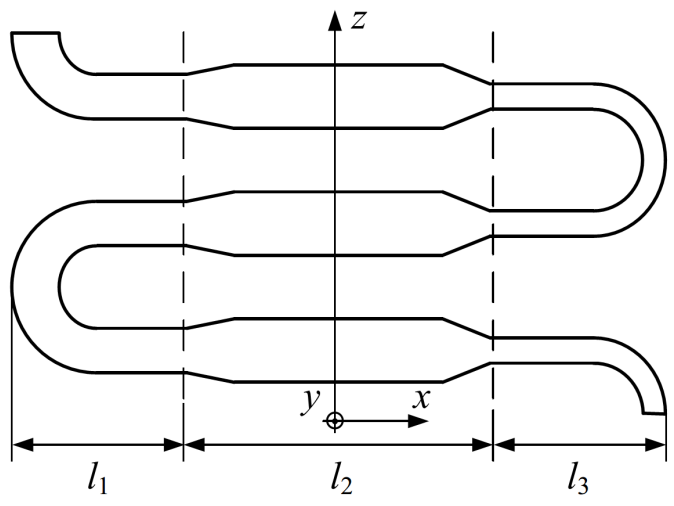

Fig. 4. The fragment of the asymmetrical meander electrode: $l_{2}$ is the central section of meander electrode, $l_{1}, l_{3}$ are the peripheral sections of meander electrode.

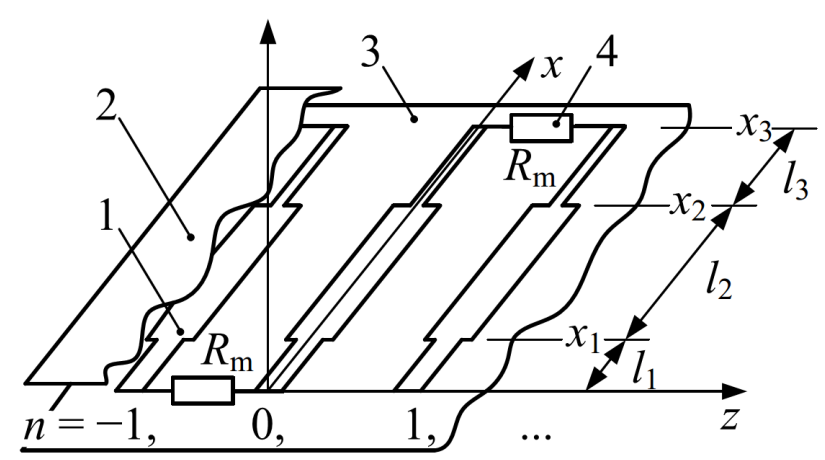

Fig. 5. The model of the meander delay line: 1 is the conductor of the multiconductor line; 2,3 are the shields; 4 is the resistance $R_{\mathrm{m}}$ in multiconductor line model that simulates certain material; $n$ is the number of conductor; $l_{1}, l_{2}, l_{3}$ are the lengths of homogeneous sections and $x_{1}, x_{2}, x_{3}$ are the coordinates of intersections of homogeneous sections in $x$ axis. 
The model of the meander delay line (MDL) containing transverse inhomogeneity is presented in Fig. 5. The model consists of three homogeneous sections of the multiconductor line. Their lengths and characteristic impedances can be different.

Expressions for voltages and currents of the conductors in the multiconductor line were discussed in [36]. The multiconductor line models the meander system if these modified boundary conditions are satisfied

$$
\begin{aligned}
& \underline{I}_{1 n}(0)\left(R_{\mathrm{m}} / 2\right)+\underline{U}_{1 n}(0)= \\
& \underline{U}_{1(n-1)}(0)+\underline{I}_{1 n}(0)\left(R_{\mathrm{m}} / 2\right), \\
& \underline{I}_{1 n}\left(x_{1}\right)=\underline{I}_{2 n}\left(x_{1}\right), \underline{U}_{1 n}\left(x_{1}\right)=\underline{U}_{2 n}\left(x_{1}\right), \\
& \underline{I}_{2 n}\left(x_{2}\right)=\underline{I}_{3 n}\left(x_{2}\right), \\
& \underline{U}_{2 n}\left(x_{2}\right)=\underline{U}_{3 n}\left(x_{2}\right), \underline{I}_{3 n}\left(x_{3}\right)=-\underline{I}_{3(n+1)}\left(x_{3}\right), \\
& \underline{I}_{3 n}\left(x_{3}\right)\left(R_{\mathrm{m}} / 2\right)+\underline{U}_{3 n}\left(x_{3}\right)= \\
& \quad \underline{U}_{3(n+1)}\left(x_{3}\right)+\underline{I}_{3(n+1)}\left(x_{3}\right)\left(R_{\mathrm{m}} / 2\right), \\
& \underline{I}_{2(n+1)}\left(x_{2}\right)=\underline{I}_{3(n+1)}\left(x_{2}\right), \\
& \underline{U}_{2(n+1)}\left(x_{2}\right)=\underline{U}_{3(n+1)}\left(x_{3}\right), \\
& \underline{I}_{1(n+1)}\left(x_{1}\right)=\underline{I}_{2(n+1)}\left(x_{1}\right), \\
& \underline{U}_{1(n+1)}\left(x_{1}\right)=\underline{U}_{2(n+1)}\left(x_{1}\right),
\end{aligned}
$$

where $R_{\mathrm{m}}$ simulates the material of the meander electrode. After derivation of the formulae the final expressions of frequency $f$, retardation factor $k_{\mathrm{R}}$ and characteristic impedances $Z_{\mathrm{C}}$ are

$$
\begin{aligned}
& f=k c_{0} / 2 \pi ; \\
& k_{\mathrm{R}}=c_{0} / v_{\mathrm{ph}}=\theta / k L ; \\
& \operatorname{Re}\left(\underline{Z}_{\mathrm{C}}(x)\right)=\frac{\underline{U}_{0}(x)}{\underline{I}_{0}(x)}=-\mathrm{j} \frac{A_{12}+A_{14}}{Y(\theta) A_{11}+Y(\theta+\pi) A_{13}},
\end{aligned}
$$

where $v_{\mathrm{ph}}$ is the phase velocity of the travelling wave, $c_{0}$ is the light velocity and $L$ is the step of the conductors of the multiconductor line, $Y(\theta)$ and $Y(\theta+\pi)$ are the characteristic admittances and $A_{11}, A_{12}, A_{13}, A_{14}$, are the coefficients of the $A$ matrix.

\subsection{Algorithm for the estimation of losses in the meander lines}

The algorithm 2 is designed to calculate the retardation factor, the input impedance and the transfer characteristics of the MDLs. The main difference of the proposed algorithm comparing to the algorithms proposed in our previous papers [36] is the use of alternative material resistance $R_{\mathrm{m}}$, which allows to evaluate the EM wave losses in the MDLs. The influence of material resistance $R_{\mathrm{m}}$ to the frequency characteristics of MDLs reveals from equations of boundary conditions (7)-(12).
Firstly, parameters of the system of MDLs are initialized in the first block: $l_{1}, l_{2}, \ldots l_{n}$ are the lengths of the sections of meander electrode; $Z_{\mathrm{C} 1}, Z_{\mathrm{C} 2} \ldots Z_{\mathrm{C} n}$ are the characteristic impedances of the meander electrode sections; $L$ is the step between neighbour conductors of the meander electrode; $R_{\mathrm{m}}$ is the alternative material resistance received in the multiconductor line model that simulates certain material and $\theta$ is the phase angle between the voltages and currents on the adjacent conductors of the multiconductor line. The number of sections of meander electrode may vary depending on the shape of meander.

Secondly, some additional parameters has to be calculated before working with the dispersion equations in the second block of the algorithm. The number of the characteristic admittances $Y_{1}(\theta), Y_{2}(\theta), \ldots Y_{n}(\theta)$ depends on the number of sections of meander electrode. It is also important to select the initial boundaries $\theta_{\min }$ and $\theta_{\max }$ in the search range of the phase angle $\theta$ before starting the calculation of dispersion equation. It is possible to miss the solution even in cases when a solution exists if the search region is selected incorrectly.

Selection of initial value or recalculation of the phase angle $\theta$ between voltages or currents on the adjacent conductors of the meander electrodes is carried out in the third block of the algorithm. $\theta$ is recalculated by the chosen step of phase angle $\Delta \theta$, which can be adjusted. The $\theta_{\min }$ value of phase angle is usually equal to zero. The $\theta_{\max }$ do not have its limit because voltages and currents equations are trigonometric functions. Resistance $R_{\mathrm{m}}$ in multiconductor line model that simulates certain material is recalculated depending on the phase angle $\theta$ in the fourth block of the algorithm. Calculation of solutions of dispersion equation begins in the fifth block of the algorithm. Calculations are carried out in an iterative process. Therefore, the number it of iterations is selected depending on the desired accuracy primarily in the fifth block. The determinant $|D|_{\mathrm{i}, \text { it,idet }}$ is calculated 3 times near the expected solution at points $\theta_{\text {min }}, \theta_{\text {average }}$, and $\theta_{\max }$ in every iteration in the sixth block. Results of calculation of three determinants $|D|_{\mathrm{i} \text {,it,idet }}$ are saved and can be used in the following stages.

Verification is performed in order to check if the solution exists in the selected range in the seventh block. Calculations move to the next iteration if there are no solutions found (third block). Narrowing of the search range is carried out if the solution exists. The narrowing is performed by recalculating boundaries $\theta_{\min }$ and $\theta_{\max }$ of the search range of the phase angle $\theta$ in the eighth block of the algorithm. The cycle is interrupted when the desired accuracy is reached which is defined by the $S_{\mathrm{al}}$ variable in the ninth block. Values of wave number are calculated according to the corresponding $\theta$ values until the desired accuracy is reached and saved. Frequency $f$, retardation factor $k_{\mathrm{R}}$, characteristic impedance $Z_{\mathrm{C}}$ are calculated when there is a known relationship between $\theta$ and wave number in the tenth block of the algorithm. New boundaries $\theta_{\min }$ and $\theta_{\max }$ of search range of the 


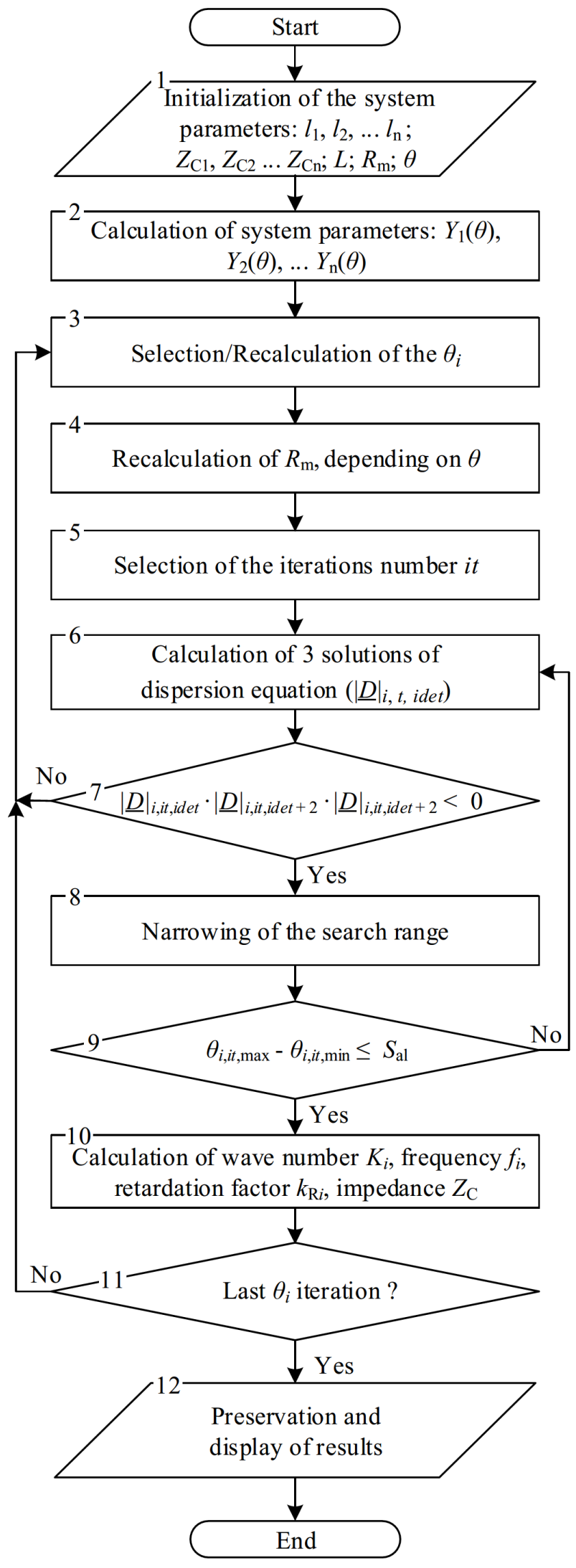

Fig. 6. The basic algorithm for the analysis of the meander delay lines (algorithm 2). phase angle $\theta$ are recalculated after return to the fourth block on condition that it is not the last iteration. Results are saved in the twelfth block if it is the last iteration of $\theta_{i}$. The calculation of frequency $f$, retardation factor $k_{\mathrm{R}}$ and characteristic impedance $Z_{\mathrm{C}}$ is performed according to Eqs. (13)-(15).

The main difference of the proposed algorithm by comparing it to the algorithms, which are proposed in our previous papers [36], occurs and are implemented in blocks 4-10. Material resistance $R_{\mathrm{m}}$ appears in equations of boundary conditions therefore it impacts the calculation of solutions of dispersion equations in the sixth block of the algorithm. The calculation of frequency, retardation factor and characteristic impedance depends on solutions of dispersion equations in the tenth block. The introduced alternative material resistance $R_{\mathrm{m}}$ allows to evaluate the EM wave losses in the MDLs. The developed algorithm could be used for the analysis of any type of meander structures containing the periodical inhomogeneities and influenced by the attenuation.

The main advantage of the second algorithm, which is proposed in this paper (see Fig. 6), by comparing it to estimation of the same parameters by commercial software, is the ability to directly estimate the dependences of the frequency and the retardation factor for the meander line. In addition, the characteristic impedance can be estimated without the need of redesign of the meander line structure or changing the mathematical model of the designed subsystem.

\section{Results}

In order to compare the loss estimation results with the alternative methods, we have applied the proposed algorithms to the microwave systems that have been investigated previously by other authors or by using the commercial software tools.

\subsection{Results of the open cylindrical waveguide analysis algorithm verification}

The model of open waveguide of ISCh4 ferrite was selected for the verification of the numerical results. The permittivity of ISCh4 ferrite was equal to $\varepsilon_{\mathrm{r}}^{\mathrm{f}}=13.5$ and the tangent of the loss angle was $\tan \delta=5 \times 10^{-4}$ and the complex relative permeability of the waveguide was equal to $\underline{\mu}_{\mathrm{r}}^{\mathrm{f}}=1-\mathrm{j} 5 \times 10^{-3}$. Dielectric material, with the complex permittivity $\varepsilon_{\mathrm{r}}^{\mathrm{d}}=9.6\left(1-\mathrm{j} \times 10^{-4}\right)$ and the relative thickness $d / r^{f}=0.3$ was selected for the external dielectric layer.

Dependences of the attenuation coefficient on the frequency of propagating $\mathrm{HE}_{11}$ mode in the ferrite-dielectric ISCh4 waveguide are presented in Fig. 7 . These results were compared by using three different methods: Muller, the mix of the different methods implemented in the commercial software CST Microwave Studio ${ }^{\circledR}$ and the algorithm proposed by Nickelson et al. [9]. From received characteristics it is seen that the highest differences between results were received between our proposed algorithm and Ref. [9]. The mean difference between results 
was received in frequency range from $35 \mathrm{GHz}$ to $65 \mathrm{GHz}$ as $9 \%$. The highest difference was $35 \%$ and it was received at $25 \mathrm{GHz}$.

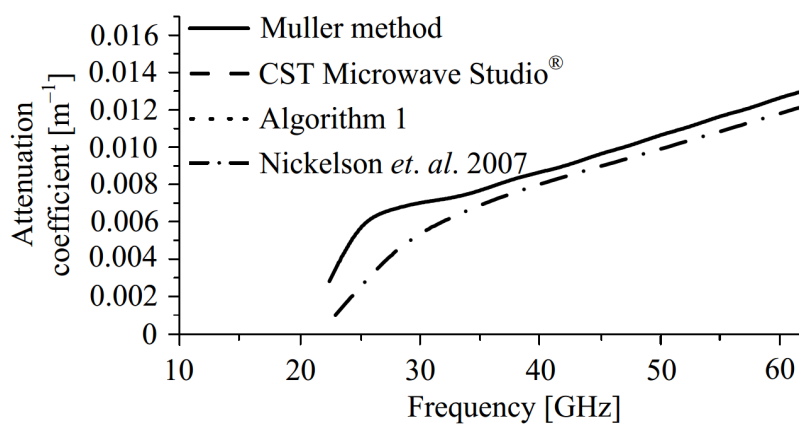

Fig. 7. Dependences of attenuation coefficient on the frequency of propagated $\mathrm{HE}_{11}$ mode in models of ferritedielectric ISCh4 waveguides.

The algorithm or method, which was used to calculate the attenuation in ferrite-dielectric ISCh4 waveguides, was not given in Nickelson et al. monograph [9]. The differences between results, received by application of the rest two algorithms were very small in all investigated frequency range and was about $\approx 0.01 \%$. These differences are not seen in dependences (Fig. 7).

$n$-GaAs semiconductor was selected for the verification of the numerical semiconductor-dielectric waveguide analysis results. Results were compared for the materials with density of electrons equal to $N=10^{19}$; $10^{20} \mathrm{~m}^{-3}$ and setting different temperature $T=125$, $150,175,200$ K. Dependences of semiconductor-dielectric waveguides where calculated when external dielectric layer relative thickness is $d / r^{\mathrm{s}}=0.3$ and EM wave frequency is $f=30 \mathrm{GHz}$. $\mathrm{Rb}_{1-x}\left(\mathrm{ND}_{4}\right) \mathrm{D}_{2} \mathrm{PO}_{4}$ dielectric was selected for external dielectric layer [35]. The differential phase shift module and attenuation constant on different temperatures of semiconductor-dielectric $n$ GaAs waveguides were compared by using the proposed algorithm 1 and the CST Microwave Studio ${ }^{\circledR}$.

Dependences of the differential phase shift module on temperature of main $\mathrm{HE}_{11}$ mode of the semiconductordielectric $n$-GaAs waveguides are presented in Fig. 8. It is seen from the characteristics (Fig. 8) that these waveguides could work as phase shifters in the range from $125 \mathrm{~K}$ to $150 \mathrm{~K}$ and in the range from $175 \mathrm{~K}$ to $200 \mathrm{~K}$. The highest differential phase shift module was received in these two ranges of the temperature. The highest differential phase shift module was received for the material with density of electrons $N=10^{20} \mathrm{~m}^{-3}$ (Fig. 8b).

It is seen from the results that the highest differences of calculated results were in temperature range from $150 \mathrm{~K}$ to $175 \mathrm{~K}$ and the difference seeks $\approx 15.2 \%$. The difference between results decreased at the highest temperature. The difference was $\approx 3.07 \%$, when temperature is $T=200 \mathrm{~K}$ and $N=10^{19} \mathrm{~m}^{-3}$ (Fig. 8a). The difference

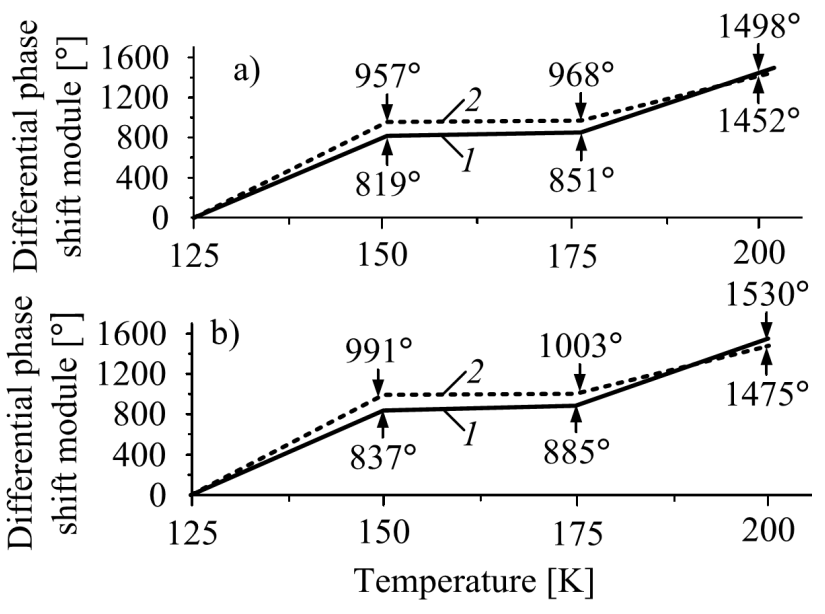

Fig. 8. Dependences of differential phase shift module on the temperature of propagated $\mathrm{HE}_{11}$ mode in models of semiconductor-dielectric $n$-GaAs waveguides: 1 algorithm 1; 2-CST Microwave Studio, where (a) $N=10^{19} \mathrm{~m}^{-3}$ (b) $-N=10^{20} \mathrm{~m}^{-3}$.

between results increased until $\approx 0.6 \%$ in temperature range from $150 \mathrm{~K}$ to $175 \mathrm{~K}$ and until $\approx 0.43 \%$, for the material with $N=10^{20} \mathrm{~m}^{-3}$ - a ten times higher density of electrons (Fig. 8b).

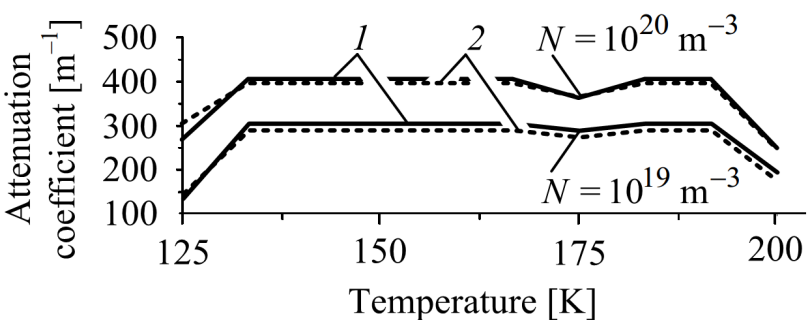

Fig. 9. Dependences of attenuation constant on the temperature of propagated $\mathrm{HE}_{11}$ mode in models of semiconductor-dielectric $n$-GaAs waveguides: 1 - algorithm 1;2-CST Microwave Studio, where $N=10^{19}$; $10^{20} \mathrm{~m}^{-3}$.

Dependences between the attenuation constant and the temperature of the main $\mathrm{HE}_{11}$ mode of semiconductor-dielectric $n$-GaAs waveguides are presented in Fig. 9. It is seen that EM wave attenuation increased when the density of electrons $N$ was higher. However, it is seen that the better way is to use the semiconductor-dielectric waveguides with lower density of electrons.

The lower difference between results was received at the temperature $T=175 \mathrm{~K}$. The difference was $\approx 5.3 \%$ in all investigation cases. However, the difference between results was the same in two temperature ranges from $137 \mathrm{~K}$ to $170 \mathrm{~K}$ and from $180 \mathrm{~K}$ to $192 \mathrm{~K}$. It means that the algorithms for the calculation of attenuation work almost similar. At the highest temperatures, the differences between results decreased to $\approx 1 \%$. 


\subsection{Results of the meander structures analysis algorithm verification}

The model of the MDL with transverse inhomogeneities was investigated in order to elucidate influence of the periodical symmetrical and asymmetrical inhomogeneities on the frequency characteristics of the meander delay system. Results of the retardation factor and input impedance versus frequency of simulation experiment of MDL regardless of losses are presented in Fig. 10.
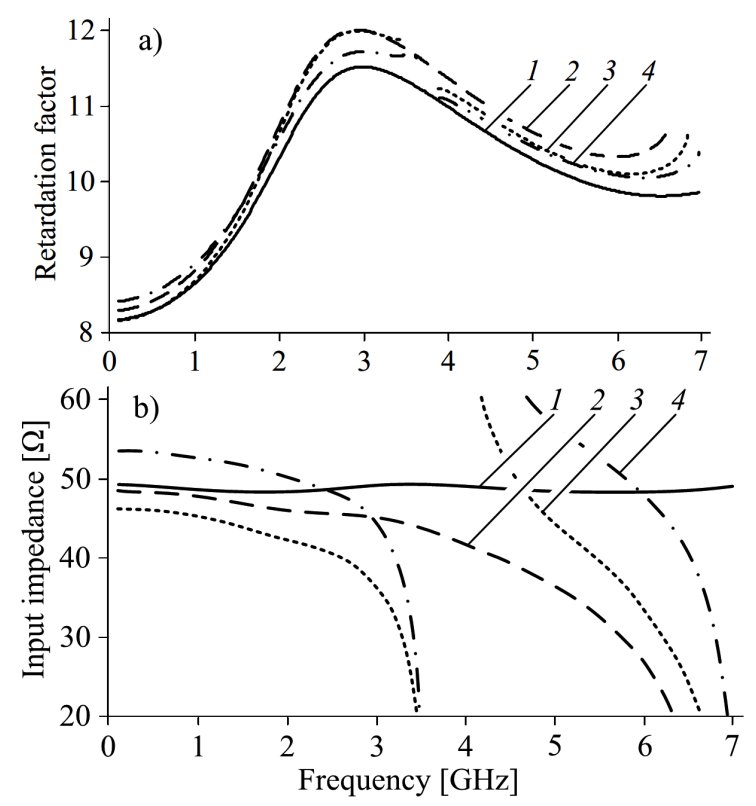

Fig. 10. Retardation factor (a) and input impedance (b) versus frequency of the inhomogeneous meander delay line at $x_{3}=l_{1}+l_{2}+l_{3}=20, L=2 \mathrm{~mm}$ and: $1-l_{1}=5 \mathrm{~mm}, l_{2}=10 \mathrm{~mm}, l_{3}=5 \mathrm{~mm}$, $Z_{1}(0) / Z_{1}(\pi)=60 / 40 \Omega, Z_{2}(0) / Z_{2}(\pi)=60 / 40 \Omega$, $Z_{3}(0) / Z_{3}(\pi)=60 / 40 \Omega ; 2-l_{1}=5 \mathrm{~mm}$, $l_{2}=10 \mathrm{~mm}, l_{3}=5 \mathrm{~mm}, Z_{1}(0) / Z_{1}(\pi)=70 / 50 \Omega$, $Z_{2}(0) / Z_{2}(\pi)=50 / 30 \Omega, Z_{3}(0) / Z_{3}(\pi)=70 / 50 \Omega ;$ $3-l_{1}=5 \mathrm{~mm}, l_{2}=10 \mathrm{~mm}, l_{3}=5 \mathrm{~mm}$, $Z_{1}(0) / Z_{1}(\pi)=70 / 50 \Omega, Z_{2}(0) / Z_{2}(\pi)=50 / 30 \Omega$, $Z_{3}(0) / Z_{3}(\pi)=60 / 40 \Omega ; 4-l_{1}=10 \mathrm{~mm}$, $l_{2}=5 \mathrm{~mm}, l_{3}=5 \mathrm{~mm}, Z_{1}(0) / Z_{1}(\pi)=50 / 50 \Omega$, $Z_{2}(0) / Z_{2}(\pi)=50 / 30 \Omega, Z_{3}(0) / Z_{3}(\pi)=70 / 50 \Omega$.

At constant characteristic impedances of the meander conductors the frequency characteristics of the MDL were continuous (curves 1 in Fig. 10a,b). Relatively great dispersion of the retardation and small variation of the input impedance were received in homogeneous MDL characteristics. These results corresponded to those, which were received in previous research work [36].

In traveling-wave deflection systems, the meander electrodes containing wide central parts of the meander conductors and narrowed the peripheral parts were used for investigation (in order to receive the high sensitivity of the cathode-ray tube and the high impedance of its signal path). Characteristics of the model of such MDL are presented by curves 2 in Fig. 10a,b. According to the received input impedance frequency characteristics (Fig. 10b), the input impedance of the system changed rapidly with increased frequency and the stop-band appeared at the phase angle $\theta$ values approached to $\pi$. The received cut-off frequency can be expressed by the following equation:

$$
f_{\mathrm{c}, \pi}=1 /\left(2 t_{\mathrm{d}}\right),
$$

where $t_{\mathrm{d}} \cong x_{3} k_{\mathrm{R}} / L c_{0}$ is the delay time corresponding to the step of meander conductors.

The remaining two curves presented in Fig. 10a,b illustrates the influence of the asymmetrical inhomogeneities on the properties of MDL. The received curves 3 were obtained at different peripheral parts of the MDL, curves 4 - at the shifted central part of the meander electrode (different lengths of peripheral parts). Comparing the characteristics 3 and 4 with the characteristic of the MDL with transverse symmetry (curves 1 and 2) it can be found that the asymmetry of the system causes radical changes of its frequency properties. At the asymmetry range the input impedance rapidly changed when the phase angle $\theta$ approached $\pi / 2$. As a result, the discontinuity of the frequency characteristics and the stop band appeared at cut-off frequency, which can be expressed by the following equation:

$$
f_{\mathrm{c}, \pi / 2}=1 /\left(4 t_{\mathrm{d}}\right) \text {. }
$$

Effects caused by the asymmetry can be explained taking into account that at the transverse asymmetry the period of the inhomogeneities along the meander conductor becomes twice greater with respect to the period of inhomogeneities at the absence of transverse asymmetry.

Results (1, 2 curves - our algorithm, 3, 4 curves Sonnet $\left.{ }^{R}\right)$ of the received retardation factor and the input impedance versus frequency of the MDL, taking into account losses in conductors when using equivalent resistance $R_{\mathrm{m}}$, are presented in Fig. 11. The verification of our algorithm was performed using the Sonnet ${ }^{\circledR}$ software package. Copper with conductivity equal to $5.8 \times 10^{7} \mathrm{~S} / \mathrm{m}$ was selected for analysis in Sonnet ${ }^{\circledR}$ programming package. Copper material was simulated by choosing the equivalent resistance $R_{\mathrm{m}}$ in our algorithm. The equivalent resistance $R_{\mathrm{m}}$ was selected to match the resistance of the copper, selected in Sonnet ${ }^{\circledR}$ package.

It is seen in Fig. 11 that the influence of losses was evident and the biggest impact of losses was received in the lower frequencies until the stop-band for the analysis results obtained by our method (see 1, 2 curves in Fig. 11). The retardation factor was higher in low frequencies and was smaller near the stop-band in system with losses. The influence of the active resistance and conductivity relatively decreased with the increased frequency. In addition, the impact of losses to the frequency characteristics of the meander line decreased. The results obtained using our method were validated using commercial Sonnet ${ }^{\circledR}$ software package (see 3, 4 curves in Fig. 11). Sonnet ${ }^{\circledR}$ uses the method of moments applied directly to Maxwell's equations to solve planar problems. Method 

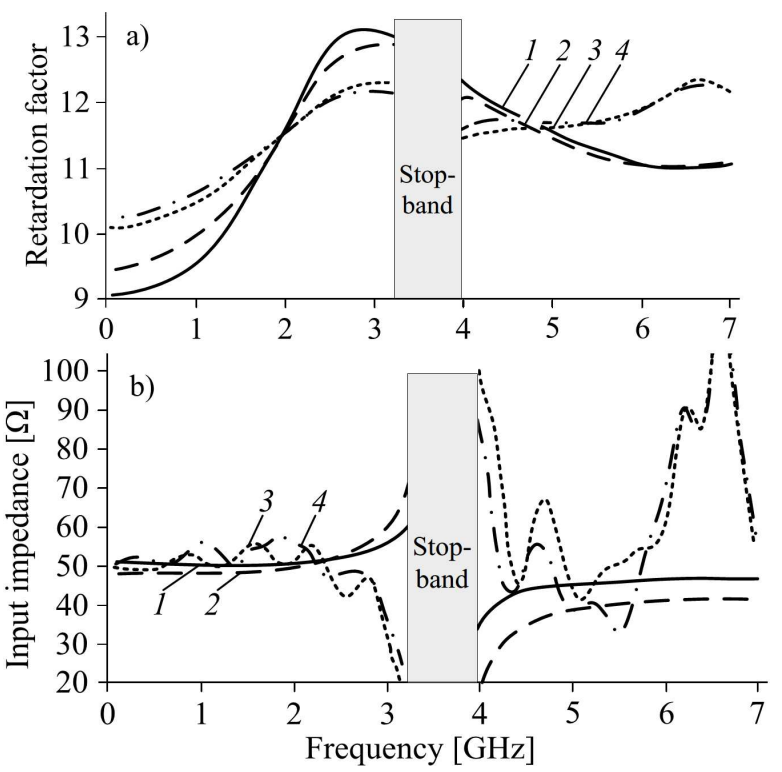

Fig. 11. Retardation factor (a) and input impedance (b) versus frequency of the inhomogeneous meander delay line at $x_{3}=l_{1}+l_{2}+l_{3}=20$, $L=2 \mathrm{~mm}$ and: 1 - Our algorithm: $l_{1}=5 \mathrm{~mm}$, $l_{2}=10 \mathrm{~mm}, l_{3}=5 \mathrm{~mm}, Z_{1}(0) / Z_{1}(\pi)=60 / 40 \Omega$, $Z_{2}(0) / Z_{2}(\pi)=60 / 40 \Omega, Z_{3}(0) / Z_{3}(\pi)=60 / 40 \Omega ; 2-$ Our algorithm: $l_{1}=5 \mathrm{~mm}, l_{2}=10 \mathrm{~mm}, l_{3}=5 \mathrm{~mm}$, $Z_{1}(0) / Z_{1}(\pi)=70 / 50 \Omega, Z_{2}(0) Z_{2}(\pi)=50 / 30 \Omega$, $Z_{3}(0) / Z_{3}(\pi)=70 / 50 \Omega$, Metal-Copper, equivalent resistance $R_{\mathrm{m}}=1 / \delta, \delta=5.8 \times 10^{7} \mathrm{~S} / \mathrm{m} ; 3$ - Sonnet: $l_{1}=5 \mathrm{~mm}, l_{2}=10 \mathrm{~mm}, l_{3}=5 \mathrm{~mm}$, metal-lossless, $\delta=\infty \mathrm{S} / \mathrm{m}$, wall thickness $=0.01 \mathrm{~mm} ; 4$ - Sonnet: $l_{1}=10 \mathrm{~mm}, l_{2}=5 \mathrm{~mm}, l_{3}=5 \mathrm{~mm}$, metal-copper, $\delta=5.8 \times 10^{7} \mathrm{~S} / \mathrm{m}$, wall thickness $=0.01 \mathrm{~mm}$.

of moments takes into account more factors, therefore, the formation of another stop-band was noticeable at the $6.5 \mathrm{GHz}$.

It should also be noted that the verification of our method was qualitative rather than quantitative. It is difficult to ensure the equal conditions for both methods, so it was noticeable that the dynamics of the frequency characteristics variation were the same, but the exact values in compared frequency characteristics did not match.

\section{Conclusions}

A comparative analysis has shown that the algorithms, proposed in this paper allows to evaluate the losses in microwave devices based on the open cylindrical waveguides or based on the meander delay lines.

The attenuation of propagated $\mathrm{HE}_{11}$ mode estimated by the proposed algorithm (designed for the waveguide-based microwave devices) for the frequency range from $10 \mathrm{GHz}$ to $66 \mathrm{GHz}$ was the same as attenuation estimated using Muller method and attenuation estimated using CST Microwave Studio ${ }^{\circledR}$.

The validation of the results was performed by comparing proposed algorithms with the commercial software tools and available alternative methods. During investigation of the open cylindrical $n$-GaAs waveguides with external $\mathrm{Rb}_{1-x}\left(\mathrm{ND}_{4}\right) \mathrm{D}_{2} \mathrm{PO}_{4}$ dielectric layer, the constant attenuation of 302.21 for the material with $N=10^{19} \mathrm{~m}^{-3}$ and the attenuation of 405 for the material with $N=10^{20} \mathrm{~m}^{-3}$ was received in temperature range from $137 \mathrm{~K}$ to $170 \mathrm{~K}$ and from $180 \mathrm{~K}$ to $192 \mathrm{~K}$. The difference between the values received by the algorithm proposed in this paper and the values received by the CST Microwave Studio ${ }^{\circledR}$ were in the range of $6 \%$.

The evaluation of losses by the second algorithm, which is proposed in this paper, should be used during the analysis of the frequency characteristics of the meander system-based microwave devices. The received results has shown that at the transverse asymmetry the period of inhomogeneities along the meander conductor becomes twice greater with respect to the period of inhomogeneities at the absence of transverse asymmetry. Moreover, the input impedance changes rapidly and the stop-band appears when phase angle $\theta$ approaches to $\pi / 2$.

Comparison of results received during the investigation of the meander delay system with losses evaluated using proposed algorithm and without evaluation of losses showed that losses have a significant impact on the frequency characteristics of the meander delay systems, especially in the comparatively low frequency range until the stop-band edge frequency of $3.2 \mathrm{GHz}$. The influence of the active resistance and the conductivity relatively decreases in higher frequencies and the impact of losses to frequency characteristics of the meander delay line decreases. The performed verification with commercial Sonnet ${ }^{\circledR}$ software package confirmed the performance of the proposed loss evaluation algorithm for the meander system-based microwave devices.

\section{References}

[1] S.M. Djurič, N.M. Djurič, M.S. Damnjanovič, J. Microelectron. Electron. Comp. Mater. 45, 2 (2015).

[2] A. Gurskas, V. Urbanavičius, R. Martavičius, Electron. Electr. Eng. 99, 3 (2010).

[3] E. Metlevskis, R. Martavičius, Electron. Electr. Eng. 119, 3 (2012).

[4] V. Urbanavičius, Š. Mikučionis, R. Martavičius, Electron. Electr. Eng. 77, 5 (2007).

[5] C. Yeh, I.F. Shimabukuro, The Essence of Dielectric Waveguides, Springer Science Business Media, New York 2008.

[6] A. Kosi, M. Solar, J. Sci. Industr. Res. 69, 569 (2010).

[7] F.M. Tang, J.C. Li, C. Li, Comput. Eng. Electron. 2, 767 (2015)

[8] S. Xie, Y. Su, C. Tang, L. Chen, WSEAS Trans. Circ. Syst. 14, 247(2015).

[9] L. Nickelson, S. Asmontas, V. Malisauskas, V. Sugurovas, The Open Cylindrical Gyrotropic Waveguides, Technika, 2007.

[10] N.R. Erickson, R.M. Grosslein, IEEE Trans. Microw. Theory Techn. 55, 2495 (2007). 
[11] S.S. Iqbal, A.A. Gibson, in: Proc. 11th Int. Conf. on Antennas and Propagation, IET, Manchester 2001, p. 323.

[12] S.S. Iqbal, Microw. Opt. Technol. Lett. 39, 4 (2003).

[13] A. Bubnelis, L. Nickelson, R. Martavicius, in: Proc. MIKON 2012, 19th Int. Conf. on Microwaves, Radar and Wireless Communications, Warsaw (Poland), Ed. R. Kubacki, IEEE, Warsaw 2012, p. 487.

[14] Y.J. Huang, W.T. Lu, S. Sridhar, Phys. Rev. A 77, 11 (2008).

[15] Q. Zhang, T. Jiang, Y. Feng, in: Proc. Microwave Conf., Ed. F. Lin, IEEE, Singapore 2009, p. 1242.

[16] H. Lin, A.I. Zaki, IEEE Trans. Magn. 25, 25 2950(1989).

[17] I.R. Belous, in: Proc. the Fourth Int. Kharkov Symp., IEEE, Kharkov 2001, p. 600.

[18] B.P. Catrysse, S. Fan, in: 21st Annual Meeting of the IEEE Lasers and Electro-Optics Society, 2008.

[19] L. Nickelson, S. Asmontas, V. Malisauskas, R. Martavicius, J. Plasma Phys. 75, 1 (2008).

[20] L. Nickelson, T. Gric, S. Asmontas, R. Martavicius, Electron. Electr. Eng., 93 87(2009).

[21] L. Nickelson, S. Asmontas, T. Gric, R. Martavicius, in: Progress in Electromagnetics Research Symp., Moscow (Russia), Electromagnetics Academy, Moscow 2009, p. 573.

[22] T. Gric, L. Nickelson, S. Asmontas, Progr. Electromagn. Res. 109, 361 (2010).

[23] J. Bucinskas, L. Nickelson, V. Shugurovas, Progr. Electromagn. Res. 105, 103 (2010).

[24] H. Oraizi, A. Abdolali, Progr. Electromagn. Res. 78, 129 (2008)
[25] J. Bucinskas, L. Nickelson, V. Shugurovas, Progr. Electromagn. Res. 109, 175 (2010).

[26] E. Kelebekler, N. Yener, in: Progress in Electromagnetics Research Symp. Proc., Marrakesh (Morocco), Marrakesh 2011, p. 1084.

[27] E. Kelebekler, Progr. Electromagn. Res. B 54, 357 (2013).

[28] S. Liu, L.W. Li, M.S. Leong, T.S. Yeo, Progr. Electromagn. Res. 29, 231 (2000).

[29] L. J. Golanka, Bull. Pol. Acad. Sci. Techn. Sci. 54, 2 (2006).

[30] P. Vikram, H.V. Kumaraswamy, R.K. Manjunath, Int. J. Adv. Res. Comput. Commun. Eng., 4 (2015).

[31] A. Gurskas, A. Krukonis, V. Urbanavicius, Electron. Electr. Eng. 21, 2 (2015).

[32] A.A. Eldek, Progr. Electromagn. Res. B 2, 177(2008).

[33] Q.W. Che, N.K.E. Yung, K. Wu, D.X. Nie, Progr. Electromagn. Res. 45, 263 (2004).

[34] D. Plonis, V. Malisauskas, A. Serackis, Acta Phys. Pol. A 119, 542 (2011).

[35] S. Štaras, R. Martavičius, J. Skudutis, V. Urbanavičius, V. Daškevičius, Wide-Band Slow-Wave Systems: Simulation and Application, CRC Press, Boca Raton 2012.

[36] A. Katkevičius, S. Štaras, Electron. Electr. Eng. 2, 108 (2011).

[37] J. Banys, A. Kajokas, S. Lapinskas, A. Brilingas, J. Grigas, J. Petzelt, S. Kamba, J. Phys. Condens. Matter 14, 14 (2002). 